\title{
Keynotes
}

\section{General practitioner fundholding and psychiatric practice}

\author{
ANDREw Sims, President, Royal College of Psychiatrists
}

This is a personal view on the implications for mental health services of the Executive Letter of the National Health Service Management Executive (NHSME), published in July 1992 (EL (92) 48): 'Guidance on the extension of the Hospital and Community Health Services elements of the GP Fundholding Scheme from Ist April, 1993'.

Some fundholding general practitioners started purchasing specialist care from 1 April 1991. Although charged for out-patient referrals to the consultant psychiatrist, referrals to clinical psychologists or community psychiatric nurses were free. The Royal College of Psychiatrists and the Joint Consultants Committee was informed by the NHSME that there was no intention to distort referral patterns but codes simply did not exist for these latter referrals. There have, however, been reports of individual general practitioners decreasing referrals to consultants and taking advantage of this "free service".

From 1 April 1993 general practice fundholders are to be charged for all referrals to mental health services and also to specialist services for people with learning disabilities. The Executive Letter states: "Secondary care mental health services are generally provided by integrated multidisciplinary mental health teams which include psychiatric nurses, social workers and, depending on local circumstances, psychologists, occupational and other therapists, psychotherapists and counsellors".

General practitioner fundholders are informed that they will need to consider how their local mental health chooses to operate. It is stated that direct referrals to individual disciplines may not be feasible and, in particular, referrals to community psychiatric nurses may not be acceptable policy in a specialist mental health service. The Executive Letter commends the Care Programme Approach (Department of Health, 1990): "Where a fundholder purchases psychiatric services from an NHS Unit or Trust, the contractual arrangement should require these organisations to operate fully the "Care Programme Approach' as specified in HC (90) 23/LASSL (90) 11".

The essential elements of the Care Programme Approach are: There should be planned community care and in particular discharge arrangements for all patients seen in the service; effective working relationships with Social Services Departments are essential; the Approach implies the appointment of a key worker. There is scope in the Executive Letter for consultants to organise their service in the way they consider appropriate: "Contracts must also take account of local clinical protocols, which may require referrals to be made to the mental health team or to the consultant, and mirror local arrangements for costing. As with community nursing services, to avoid any misunderstanding and disputes, it is important that in the contracting process a clear understanding is developed of the services to be covered. In setting their prices, mental health units may base their charges on average specialty costs, in which case a single price would be applied irrespective of the service provided by the team. In view of the wide variation which can exist in the number of attendances within one psychiatric episode, providers should be encouraged to introduce scales and charges based on either a rate per attendance/ visit or on banding numbers of attendances in ranges".

The document recognises "the need to concentrate skilled psychiatric care on the more severely mentally ill"... "Although some NHS providers will be able to offer direct access to some of the mental health services individually (e.g. community psychiatric nursing), this will not always be feasible and fundholders will need to bear this in mind in their discussions with providers. CPNs are a scarce resource in some areas and unless mental health units are in a position to respond to additional demand, offering additional separate CPN services to fundholders could jeopardize the delivery of a comprehensive mental health service to the seriously mentally ill. Where fundholders wish to purchase additional resources for the treatment of the less severely mentally ill who may not require the input of the whole team, they may wish to consider the contribution which can be made by counsellors working within the primary health care team. Counselling is therefore being added to the list of services which they may purchase". 
The profession of community psychiatric nursing was originally established to look after the severely mentally ill, especially those suffering from chronic schizophrenia in the community. However the recent Third Quinquennial Report on Community Psychiatric Nursing (White, 1991) has shown that only $17 \%$ of those CPNs who specialise, do so in rehabilitation; that is only $7 \%$ of all CPNs specialise in rehabilitation. About half the referrals to CPNs now come from general practitioners. Only about half of the patients who currently form the CPN case-load have had previous psychiatric admission; $43 \%$ are chronically mentally ill; and $27 \%$ suffer from schizophrenia. From data made available by the National Schizophrenia Fellowship in 1990 , $81 \%$ of schizophrenic patients in the community currently receive no care from a CPN; $23 \%$ of CPNs have no schizophrenic patients at all on their case load.

There has been a three-fold increase in the number of CPNs over the last decade. With current members, both of CPNs and schizophrenic patients in the community, caring for these patients alone could produce an adequate case-load for each CPN. In an average general practice of 2,000 people with perhaps seven schizophrenic patients, at a conservative estimate there might be 400 showing 'conspicuous psychiatric morbidity'. If many such patients are directly referred to CPNs, the nurse will make almost no impact on general practice psychiatric morbidity but will have no time available for the severely ill. CPN training, and their range of available treatments, does not equip them to deliver effective treatment for much of the general practice psychiatric morbidity. Unfortunately, unsuccessful and at times inadequate treatment may reduce the confidence of patients and their relatives in psychiatry, and may hence increase the stigma of mental illness. Prolonged intervention may for some reinforce the sick role and create dependence. At the same time this use of CPN time prevents the highly effective care which nurses can provide for the severely mentally ill in the community.

The practice of direct referral from general practitioner to community psychiatric nurse, compounded by the service having been a 'free good' between April 1991 and 1993, and also by the fact that there is no additional remuneration to general practitioners for any aspect of treatment of the mentally ill, must represent a temptation for GPs to refer consistent complainers. As a result, the CPN service is at present in part a squandered resource, and this is a major financial reason for the failure of community care.

Council of the Royal College of Psychiatrists has recently produced a report, Mental Health of the Nation: The Contribution of Psychiatry (1992). Among other issues this deals with organisation of clinical services. It is argued that if consultant psychiatrists were enabled better to organise individual services for their patients, they could provide an improved service, possibly at less cost. A necessary component would be direct referral from the general practitioner to the consultant psychiatrist.

\section{Comment}

The Executive Letter, 'Guidance on the Extension of the Hospital and Community Health Services Element of the GP Fundholding Scheme' presents challenges and possibilities for improved psychiatric practice. A major concern will be how to preserve quality, for instance the use of counsellors in primary health care is recommended but there are at present no universal training standards or quality controls. The document concentrates on the delivery of specialist services and it is evident that clear clinical protocols will be required. Many community psychiatric services are now sectorised and the implications of this will need to be worked out in detail with general practice purchasers. Psychotherapy is referred to without comment in the Executive Letter; there is a need to delineate the essentials of a District service (Royal College of Psychiatrists, 1991). There is a need for primary care workers to have further training in dealing with the mentally ill.

The Government Strategic Report The Health of the Nation (1992) places mental illness as one of its five key areas and gives three targets to be achieved:

(a) to improve significantly the health and social functioning of mentally ill people

(b) to reduce the overall suicide rate by at least $15 \%$ by the year 2000

(c) to reduce the suicide rate of severely mentally ill people by at least $33 \%$ by the year 2000 .

Fundholding general practitioners will need to assess their contribution for achieving these targets and this should inform their referral to specialist services. At the same time specialist services should realize that general practitioners require a rapid service for new patients to receive appointments; they are concerned about the grade of doctor who sees patients, preferring a consultant; and swift communication between consultant and general practitioner throughout the episode of treatment is needed.

For effective organisation of services, it is recommended that all new referrals from the general practitioner to specialist mental health services should be made to the consultant, andgroups of consultants working together in Trusts or Units are advised to make this explicit in the protocols prepared for purchasers. The consultant psychiatrist with the multidisciplinary 
team should use the Care Programme Approach, deciding on the appropriate key worker for each referred patient. Follow-up of discharged patients from specialist care should be carefully planned and might involve consultant, general practitioner and community psychiatric nurse seeing the patient by arrangement on different occasions. Specialist mental health services both in hospital and in the community, should concentrate on the severely mentally ill, and services should only be extended to others when the needs of this group have been met. Purchasers, in order to achieve the targets of the Health of the Nation for the severely mentally ill, will need to maximise the effectiveness of mental health professionals; this implies concentrating the work of CPNs within the specialist multidisciplinary team upon caring for the severely mentally ill.

\section{References}

Departiment of Health (1990) Care Programme Approach Circular HC(90)23/LASSL(90)11. London: Department of Health.

National Health Service Management Executive (1992) Guidance on the extension of the Hospital and Community Health Services elements of the GP fundholding scheme from 1 April 1993 EL(92)48. London: Health Care Directorate.

Royal College of Psychintrusts (1991) The future of psychotherapy services. Psychiatric Bulletin, 15, 174-179. - (1992) Mental Health of the Nation: The Contribution of Psychiatrists. London: Royal College of Psychiatrists.

Secretary OF State for Health (1992) The Health of the Nation: A Strategy for Health in England 1986. London: HMSO

WhITE, E. (1991) The Third Quinquennial National Community Psychiatric Nursing Survey. Leeds CPMA Publications.

\title{
Read codes for the mental health professions: the Clinical Terms Project
}

\author{
JoHN WING, Director, College Research Unit
}

\section{An essential first step}

In mid-1991, the Conference of Medical Royal Colleges and their Faculties initiated an Advisory Group on Information Services with broad representation from the profession, the NHS Management Executive, and the Department of Health. The aim of the group is to facilitate and co-ordinate input from the profession to the NHS Information and Technology Strategy. It is chaired by Sir Duncan Nichol, NHS Chief Executive.

It was agreed that an essential first step towards building a more clinically useful Health Information System would be to establish an agreed nomenclature, or thesaurus, of terms at present used in NHS clinical records, each one with a unique identifier (the Read Code). Proposals for what has become the Clinical Terms Project, made by Dr Martin Severs, Chairman of the Conference Information Group, and Dr James Read, Director of the NHS Centre for Coding and Classification (CCC), were approved in March 1992.

The aims of the project are very simple, to provide: terms representing preferred clinical concepts agreed synonyms and eponyms for these terms agreed abbreviations

an agreed structure for convenient search and display.

It does not aim to provide:

a glossary or dictionary of differential definitions

a minimum clinical data set

a set of outcome measures

a functioning clinical information system.

The project will, however, build a platform from which such further projects can be taken forward with confidence that compatibility can be achieved throughout the NHS. This first stage will be completed by 1 October 1993.

\section{Method of working}

The majority of the work is being done by 40 Specialty Working Groups (SWGs) covering the NHS, each of which develops the section of terminology for which it is responsible while maintaining close liaison with overlapping fields. There is an elaborate quality control procedure. Representatives of all $\mathbf{4 0}$ meet at regular intervals at the CCC headquarters in Loughborough, where there is an 